Chronic Obstructive Pulmonary Diseases: Journal of the COPD Foundation

\author{
Original Research
}

\title{
Stability of Frequency of Severe Chronic Obstructive Pulmonary Disease Exacerbations and Health Care Utilization in Clinical Populations
}

Denitza P. Blagev, $\mathrm{MD}^{1,2}$ Dave S. Collingridge, $\mathrm{PhD}^{1,3}$ Susan Rea, $\mathrm{PhD}^{3,4}$ Valerie G. Press, $\mathrm{MD}, \mathrm{MPH}^{5}$ Matthew M. Churpek, MD, MPH, $\mathrm{PhD}^{5}$ Kyle Carey, $\mathrm{MPH}^{5}$ Richard A. Mularski, MD, MSHS, MCR ${ }^{6,7}$ Siyang Zeng, $\mathrm{MS}^{8,9}$ Mehrdad Arjomandi, $\mathrm{MD}^{8,9}$

\section{Abstract}

Rationale: Although chronic obstructive pulmonary disease (COPD) exacerbation frequency is stable in research cohorts, whether severe COPD exacerbation frequency can be used to identify patients at high risk for future severe COPD exacerbations and/or mortality is unknown.

Methods: Severe COPD exacerbation frequency stability was determined in 3 distinct clinical cohorts. A total of 17,450 patients with COPD in Intermountain Healthcare were categorized based on the number of severe COPD exacerbations per year. We determined whether exacerbation frequency was stable and whether it predicted mortality. These findings were validated in 83,134 patients from the U.S. Veterans Affairs (VA) nationwide health care system and 3326 patients from the University of Chicago Medicine health system.

Results: In the Intermountain Healthcare cohort, the majority (84\%, 14,706 patients) had no exacerbations in 2009 and were likely to remain non-exacerbators with a significantly lower 6-year mortality compared with frequent exacerbators ( 2 or more exacerbations per year) ( $25 \%$ versus $57 \%, p<0.001$ ). Similar findings were noted in the VA health system and the University of Chicago Medicine health system. Non-exacerbators were likely to remain non- exacerbators with the lowest overall mortality. In all cohorts, frequent exacerbator was not a stable phenotype until patients had at least 2 consecutive years of frequent exacerbations. COPD exacerbation frequency predicted any cause mortality.

Conclusions: In clinical datasets across different organizations, severe COPD exacerbation frequency was stable after at least 2 consecutive years of frequent exacerbations. Thus, severe COPD exacerbation frequency identifies patients across a health care system at high risk for future COPD-related health care utilization and overall mortality.

Abbreviations: chronic obstructive pulmonary disease, COPD; Veterans Affairs, VA; Evaluation of COPD Longitudinally to Identify Predictive Surrogate End-points, ECLIPSE; SubPopulations and InteRmediate Outcome Measures In COPD Study, SPIROMICS; emergency department, ED; international classification of diseases, $9^{\text {th }}$ revision, ICD-9; Charlson Comorbidity Index, CCI; type 2 diabetes mellitus, DMII; congestive heart failure, CHF; pulmonary function tests, PFT

Funding Support: This work was supported by the Intermountain Research and Medical Foundation (Denitza Blagev) and the Flight Attendant Medical Research Institute (Mehrdad Arjomandi). Valerie Press is supported by a K23 grant (HL118151) from the National Heart, Lung, and Blood Institute and Matthew Churpek is supported by a K08 grant (HL 121080) from the National Heart Lung and Blood Institute and R01 (GM123193).

Date of Acceptance: March 30, 2018

Citation: Blagev DP, Collingridge DS, Rea S, et al. Stability of frequency of severe chronic obstructive pulmonary disease exacerbations and health care utilization in clinical populations. Chronic Obstr Pulm Dis. 2018;5(3):208-220. doi: https://doi.org/10.15326/ jcopdf.5.3.2017.0183

\section{This article contains an online supplement.}


1 Division of Pulmonary and Critical Care Medicine, Department of Medicine, Intermountain Medical Center, Murray, Utah

2 Division of Respiratory, Critical Care, and Sleep Medicine, Department of Medicine, University of Utah, Salt Lake City

3 Office of Research, Intermountain Healthcare, Salt Lake City, Utah

4 Homer Warner Center for Informatics Research, Murray, Utah

5 Section of General Internal Medicine, Department of Medicine, University of Chicago Medicine, Chicago, Illinois

6 Kaiser Permanente Center for Health Research - Northwest, Portland, Oregon

7 Division of Pulmonary and Critical Care Medicine, Oregon Health and Science University, Portland

8 Division of Pulmonary and Critical Care Medicine, San Francisco Veterans Affairs Medical Center, San Francisco, California

9 Division of Pulmonary, Critical Care Allergy, Immunology and Sleep Medicine, Department of Medicine, University of California, San Francisco

\section{Address correspondence to:}

Denitza P. Blagev, MD

Intermountain Medical Center

Heart Lung Building, $6^{\text {th }}$ Floor

5121 South Cottonwood Street

Murray, UT 84157

Email: denitza.blagev@imail.org

denitza.blagev@gmail.com

Phone: (801) 5074695

\section{Keywords:}

chronic obstructive pulmonary disease; COPD; COPD

exacerbation; stability; health care utilization

\section{Introduction}

Chronic obstructive pulmonary disease (COPD) is characterized by lung function decline and periods of exacerbation. ${ }^{1}$ COPD exacerbations are a major cause of morbidity and mortality in COPD, and a major contributor to COPD costs. ${ }^{2-5}$ Identifying patients with COPD who are at greater risk of being hospitalized for COPD exacerbations could help in the development of targeted preventive strategies.

Several large multi-centered observational cohort studies have described the natural history of COPD and its associated exacerbations. ${ }^{3,6-8}$ These studies have included a large number of patients recruited through various methods to strengthen their applicability to the general population. However, the prevalence and incidence reports have been dissimilar in these studies. ${ }^{3,6-9}$ Data from the Evaluation of COPD Longitudinally to Identify Predictive Surrogate End-points (ECLIPSE) study described the frequent exacerbator phenotype and its stability, while data from the SubPopulations and InteRmediate Outcome Measures In COPD Study (SPIROMICS) cohort showed that 2 years of frequent exacerbations were needed before stability could be considered. ${ }^{6}$ Since the definition of an acute exacerbation of COPD (AECOPD) is a functional one (whether a patient is treated for a COPD exacerbation), whether COPD exacerbation frequency is a stable phenotype of COPD patients may vary depending on the cohort evaluated. $^{3,6}$

Thus, it remains unclear how generalizable the observed data from these research cohorts are to a clinical population of COPD patients where COPD may not be confirmed by spirometry and there is clinician variation in diagnosis. Despite this uncertainty, health care systems and clinicians are trying to identify patients at high risk for health care utilization and severe COPD exacerbations in order to target interventions aimed at reducing COPD admissions and mortality. Whether frequent severe COPD exacerbations, i.e., those requiring hospitalizations and/or emergency department (ED) visits, are stable characteristics over time in an unselected clinical population with variable comorbidities and diagnostic uncertainty remains unknown.

In this study, we retrospectively examined the prevalence, incidence, and stability of severe COPD exacerbations in patients with a diagnosis of COPD across 3 different health care systems. Our goal was to determine whether severe frequent exacerbations are a stable phenotype in this patient population, and whether severe COPD exacerbation frequency correlates with all-cause mortality.

\section{Methods}

The institutional internal review boards at Intermountain Healthcare, the University of CaliforniaSan Francisco, the San Francisco Veterans Affairs (VA) Medical Center Research and Development Committee, and the University of Chicago Medicine health system approved this retrospective data-only study and waived individual informed consent.

\section{Study Populations}

Intermountain Healthcare

We identified 17,450 living adult patients with COPD (age 18 and older in 2009) who had a face-to-face 
inpatient, ED, or ambulatory health care encounter at Intermountain Healthcare by 2013. We included patients with billing codes for COPD: international classification of diseases, ninth revision-(ICD-9) codes $(491.2,492)$ in any sequence or a DRG (190192) and their associated clinical variables, health care utilization, and demographics for each year between 2009 and 2014. Intermountain Healthcare is an integrated health system of 28 hospitals and clinics serving a large population of patients in Utah and several surrounding states. It is a relatively closed system with a large, rural population and relatively little migration. COPD was defined by billing codes. A complete list of the billing codes used to identify COPD patients across the different cohorts is presented in the online supplement Table 1.

Data for comorbidities, utilization and mortality were extracted from registration, electronic health records (EHRs), medical coding and state and national death registry systems. Charlson comorbidities are generated for each patient from all ambulatory, hospital and ED discharge diagnosis codes in our EHR and registration systems. These are updated weekly in the Analytic Health Repository as diagnosis data are refreshed. Charlson comorbidities are classified according to the validated table of ICD-10 and enhanced ICD-9 codes. $^{10}$

\section{U.S. Veterans Affairs Nationwide Health Care System} COPD exacerbation frequency and mortality were assessed in a second cohort of 83,134 patients with a COPD diagnosis from the U.S. VA nationwide health care system EHR data (VA Informatics and Computing Infrastructure, VINCI). ${ }^{11}$ Patients alive in 2009 with a diagnosis of COPD (by ICD-9 or ICD-10 codes) and laboratory values (complete blood count and complete metabolic panel in 2009) were included in the VA cohort (online supplement, Figure 1). The VA nationwide health care system is a large, integrated health system that is relatively closed.

University of Chicago Medicine Health System

A third cohort of patients from the University of Chicago Medicine health system was also analyzed. The University of Chicago Medicine health system is a small system that serves an urban population in Chicago, which is relatively open with a high rate of patients seeking care at multiple locations outside the system. A total of 3236 patients were included in the cohort after their first COPD exacerbation hospitalization between November 1, 2008 and January 1, 2016 (online supplement Figures 1 and 2 and online supplement Table 4). For this cohort, years were based on time since the index COPD exacerbation that qualified the patient for the cohort, rather than by calendar years.

\section{Study Outcomes}

The primary study outcome was to determine whether severe COPD exacerbation frequency (as defined by hospitalizations and/or ED visits for COPD exacerbation) is a stable characteristic over time. We also sought to determine whether health care utilization (any cause hospitalizations and/or ED visits) was stable over time. The secondary outcome was to determine whether health care utilization (COPD and/ or any cause) was associated with mortality differences in these cohorts.

\section{Any Cause Health Care Utilization}

We hypothesized that there may be diagnostic uncertainty in patients with a history of congestive heart failure, pneumonia, or other comorbidities and COPD when they present with respiratory symptoms. Therefore, we explored whether any cause health care utilization was more stable and predicted overall mortality in this patient population.

For the Intermountain cohort and the VA national health system cohort we had any cause hospitalization and/or ED visit data. Thus, we repeated the analysis dividing these 2 cohorts of patients based on the number of annual any cause hospitalizations and/or ED visits. Patients were divided into groups of frequent ( 2 or more visits for any cause per year), intermediate ( 1 visit for any cause per year) and infrequent (no visits for any cause per year) health care utilization.

\section{Statistical Analysis}

For each year from 2009 to 2014, we categorized patients as frequent ( 2 or more exacerbations per year), intermediate ( 1 exacerbation per year), or nonexacerbators (no exacerbations per year). We similarly categorized patient utilization of health care as frequent ( 2 or more visits for any reason), intermediate (1 visit for any reason), or infrequent (no visit for any reason). We also tracked patient any-cause mortality over the same time period.

To evaluate our primary outcome (i.e., stability of 
COPD exacerbation frequency over time), we assigned patients to a category based on the number of COPD exacerbations in 2009, then tracked those same patients in subsequent years. By arranging the bar graphs into a single figure, we were able to assess COPD exacerbation frequency stability from 2009 to 2014 using standard methods for reporting COPD exacerbation phenotype stability. ${ }^{3,6}$ Separate multi-year figures were created for COPD exacerbation and health care utilization. The COPD exacerbation frequency figure allowed us to determine whether patients who were frequent exacerbators in 2009 remained frequent exacerbators, and whether intermediate or non-exacerbators were likely to stay in those categories. The health care utilization figure allowed us to determine whether patients who were frequent utilizers of health care in 2009 remained frequent utilizers, and whether intermediate or infrequent utilizers were likely to stay in those categories.

For our secondary analysis, we used Kaplan-Meier survival analysis to evaluate the association between exacerbation frequency and health care utilization categorization in 2009, and 6-year all-cause mortality. Mortality in 6 years (yes versus no) was entered as the state variable and number of years to death was entered as the time variable. Patients who survived from 2009 to 2014 were censored. For the Intermountain Healthcare survival data, pooled over strata log-rank tests were used to compare the overall equality of the survival curves. This analysis allowed us to combine categorization levels into a single test to determine whether mortality generally differed between the 3 stability categorizations. We also ran pairwise log rank tests on the Intermountain survival data to compare individual survival distributions. Our statistical plan is summarized in a flow diagram "Flow Diagram of Statistical Analysis Plan" (online supplement).

\section{Results}

\section{COPD Exacerbation Frequency Stability and All-Cause Mortality}

Intermountain Healthcare Cohort

The Intermountain cohort comprised 17,450 adult living patients in 2009 with a prior COPD diagnosis by billing codes. Although we included all adult (age 18 and older) patients in the cohort, only $6 \%$ of patients (959 of 17,450) were under age 40 (online supplement Table 2). The baseline characteristics are summarized in Table 1.
The majority of patients $(84 \%, 14,706)$ had no exacerbations in 2009. Patients who were nonexacerbators in 2009 were likely to remain nonexacerbators between 2010 and 2014 (Figure 1). There was less stability in patients with 2 or more exacerbations in 2009. The majority of patients who were frequent exacerbators in 2009 were nonexacerbators in subsequent years (Figure 1). However, if patients were frequent exacerbators for 2 consecutive years (2009 and 2010), they were more likely to remain frequent exacerbators throughout the remainder of the study (Figures 1 and 2 ). Among patients having 2 or more exacerbations in 2009 and 2010, 32\% had 2 or more exacerbations in 2011. The percentage of patients having 2 or more exacerbations increased to $47 \%$ in $2012,44 \%$ in 2013 , and $57 \%$ in 2014 .

Overall pooled log rank tests were significant for both the COPD visit $(p<0.001)$ and any visit $(p<0.001)$ survival analyses. Statistically significant pooled log rank tests suggest that the survival curves in each analysis are different. The Chi-Square value for the COPD visit (Figure 2a) pooled log rank test is 943.45 while the Chi-Square value for the any visit (Figure $3 \mathrm{a}$ ) pooled log rank test is 520.24. A much higher COPD visit Chi-Square value (943.45) indicates that the spread among COPD visit survival curves is greater than the spread among any visit survival curves. This finding suggests that frequency of COPD visits more effectively separates cases into disparate mortality probability groups than frequency of any visits.

Frequent exacerbators in 2009 had a significantly higher probability of all-cause mortality over 6 years compared to intermediate (pairwise log rank test results, $p<0.001)$ and non-exacerbators $(p<0.001)$ (Figure 2A). Notwithstanding these differences, the 6-year mortality rate was relatively high for all patients. Among those with no hospitalizations for COPD in 2009, 24\% died over the 6-year period. Six-year mortality increased to $43 \%$ for patients with 1 exacerbation, and to $56 \%$ for those with 2 or more exacerbations (Figure 2). These high rates of mortality underscore the seriousness of a COPD diagnosis. Similar mortality rates have been found in previous studies. ${ }^{5,12}$

Frequent exacerbators had higher Charlson comorbidity rates for a variety of comorbidities (Figure 4). For example, the overall rate of cardiovascular comorbidity was high (24.0\% had diabetes and $24 \%$ had congestive heart failure [CHF]). 


\section{Table 1. Basic Demographics for the Intermountain Health Care COPD Cohort}

\begin{tabular}{|c|c|c|c|c|c|}
\hline Variable & $\begin{array}{c}\text { Overall } \\
\mathrm{N}=17,450\end{array}$ & $\begin{array}{c}\text { Non-exacerbators } \\
\text { in } 2009 \\
N=14,707\end{array}$ & $\begin{array}{c}\text { Intermediate } \\
\text { in } 2009 \\
N=2212\end{array}$ & $\begin{array}{c}\text { Frequent } \\
\text { in } 2009 \\
N=531\end{array}$ & PValue \\
\hline $\mathbf{N}(\%)$ & $17450(100.0 \%)$ & 14707 (84.3\%) & $2212(12.7 \%)$ & $531(3.0 \%)$ & $<0.001$ \\
\hline $\begin{array}{l}\text { Age in Years } \\
(\text { mean }+/- \text { std dev })\end{array}$ & $62.9 \pm 14$ & $62.6 \pm 14$ & $64.8 \pm 14$ & $66.2 \pm 13.1$ & $<0.001$ \\
\hline $\operatorname{Sex}(F, \%)$ & $8334(47.8 \%)$ & 7016 (47.7\%) & 1060 (47.9\%) & 258 (48.6\%) & 0.91 \\
\hline 6-year Mortality & $4741(27.2 \%)$ & $3484(23.7) \%$ & $958(43.3 \%)$ & 299 (56.3\%) & $<0.001$ \\
\hline CCI CHF & $4152(23.8 \%)$ & $2951(20.1 \%)$ & $887(40.1 \%)$ & $314(59.1 \%)$ & $<0.001$ \\
\hline CCI DIMII & $4187(24.0 \%)$ & $3245(22.1 \%)$ & $715(32.3 \%)$ & 227 (42.7\%) & $<0.001$ \\
\hline CCI Renal Failure & 1813 (10.4\%) & $1270(8.6 \%)$ & $409(18.5 \%)$ & $134(25.2 \%)$ & $<0.001$ \\
\hline CCI Malignancy & $2170(12.4 \%)$ & $1631(11.1 \%)$ & $418(18.9 \%)$ & $121(22.8 \%)$ & $<0.001$ \\
\hline PFT Data & 1206 (6.9\%) & 1009 (6.9\%) & $153(6.9 \%)$ & $44(8.3 \%)$ & 0.45 \\
\hline
\end{tabular}

$\mathrm{COPD}=$ chronic obstructive pulmonary disease; $\mathrm{CCI}=\mathrm{Charlson}$ Comorbidity index; $\mathrm{CHF}=$ congestive heart failure; DMII=type 2 diabetes mellitus; PFT=pulmonary function tests

Comorbidity rates increased significantly for frequent exacerbators compared to non-exacerbators (22\% versus $43 \%$ for diabetes and $20 \%$ versus $59 \%$ for $\mathrm{CHF}$, respectively, $p<0.001)$.

\section{Veterans Affairs National Health System Cohort}

Severe COPD exacerbation frequency stability and mortality were determined in 83,134 patients from the VA nationwide health care system alive in 2009 with laboratory values and an exacerbation frequency assignment (online supplement Figure 1 and online supplement Table 3). Overall, the majority of patients were non-exacerbators at baseline and remained nonexacerbators over subsequent years (Figure 5). Nonexacerbators also had a statistically significantly lower all-cause 7-year mortality as compared with intermediate or non-exacerbators (Figure 2B). Frequent exacerbators were likely to remain frequent exacerbators after 2 consecutive years of frequent exacerbations (Figure 5) and had the highest overall mortality (Figure 2B).

University of Chicago Medicine Health System Cohort Similar to the other larger cohorts, non-exacerbators were likely to remain non-exacerbators over subsequent years (Figure 6). In this small cohort there was a trend toward lower mortality in non- exacerbators compared with frequent exacerbators (Figure 7) but it did not reach statistical significance (Figure 7 and online supplement Table 5). In this cohort, of a small number of patients in a relatively open system, frequent exacerbations were not a stable phenotype until patients had 4 consecutive years of frequent exacerbations (Figure 6). However, after 2 consecutive years of frequent exacerbations, patients were more likely to have 2 or more exacerbations the subsequent year than to have none. In this relatively open system it is likely that a subset of the patients with "no exacerbations" in a given year were actually hospitalized outside the system.

\section{Any Cause Health Care Utilization Frequency Stability and Mortality}

Infrequent health care utilization was a stable phenotype identifying the lowest risk for future health care utilization and lowest mortality across both cohorts (Figures 8, 9 and 3). Frequent health care utilization in year 1 predicted subsequent year frequent health care utilization in both study cohorts (Figures 8 and 9). Additionally, frequent health care utilization was associated with a statistically significant higher overall mortality compared with infrequent health care utilization $(p<0.001$, Figure 3$)$.

For the larger cohorts, the VA and Intermountain 
Figure 1. Severe COPD Exacerbation Frequency Stability Intermountain

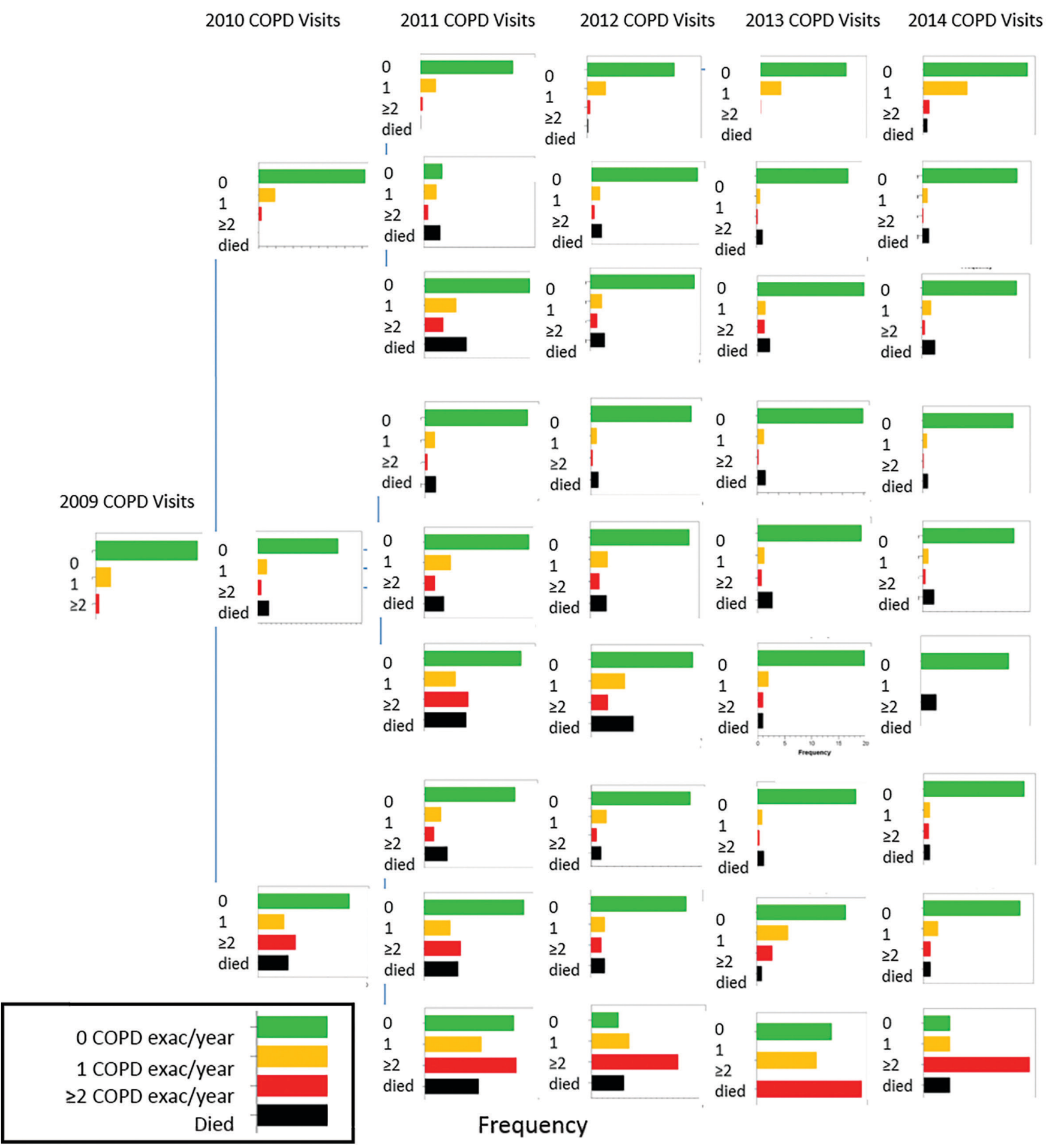

COPD exacerbation frequency for the Intermountain Healthcare cohort is shown over 6 years. Patients are divided into frequent (2 or more exacerbations per year), intermediate (1 exacerbation per year) and non-exacerbators (no exacerbations per year) in 2009 and then followed until 2014. While frequent exacerbators have a higher mortality than those with no exacerbations, exacerbation frequency becomes stable after 2 consecutive years. 


\section{Figure 2. Kaplan-Meier Survival by Severe COPD Exacerbation Frequency}

A.

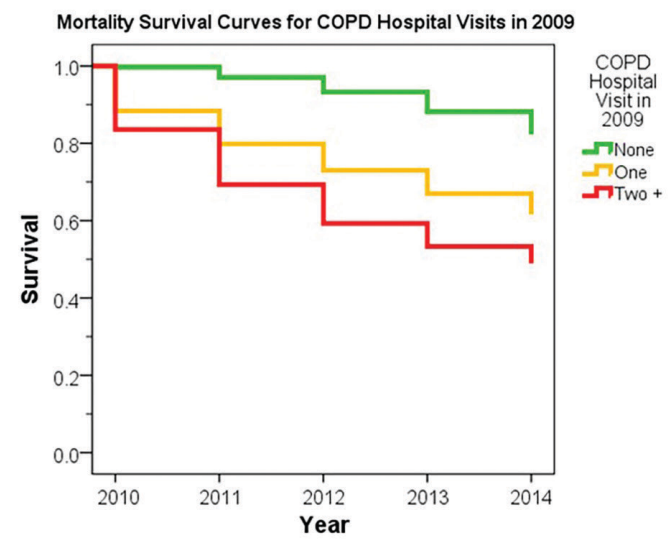

B.

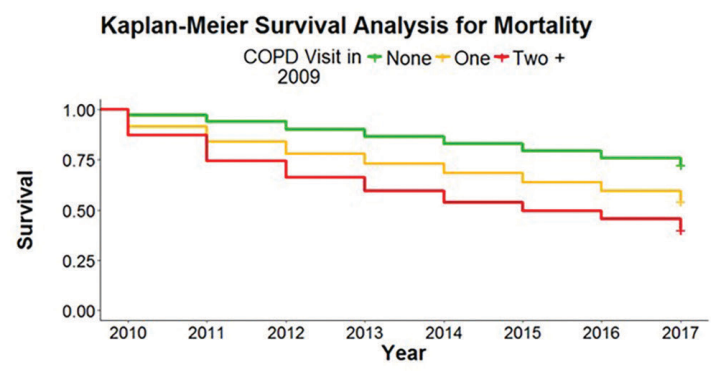

Figure 2 shows the Kaplan-Meier Survival Analysis between 2009 and 2014 based on COPD exacerbation frequency in the Intermountain cohort (Figure 4A). Panel B shows the 8-year mortality for the VA cohort based on COPD exacerbation frequency. The mortality curves separate for patients with no COPD exacerbations versus those with 1 versus those with 2 or more $(p<0.001)$.

\section{Figure 3. Kaplan-Meier Survival by Any Cause Health Care Utilization Frequency}

A.

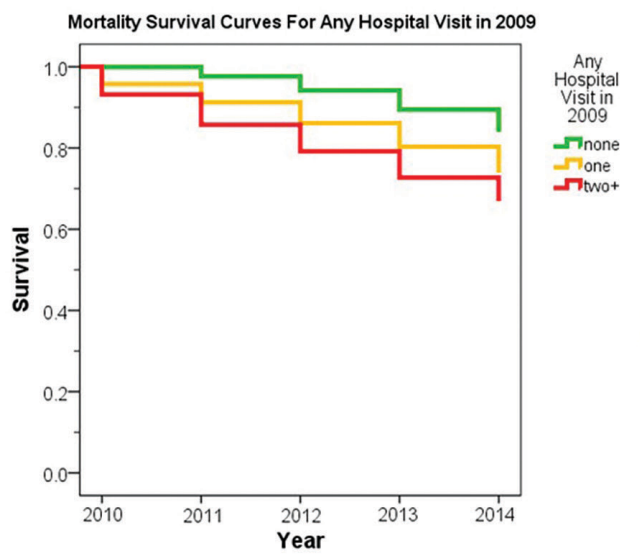

B.

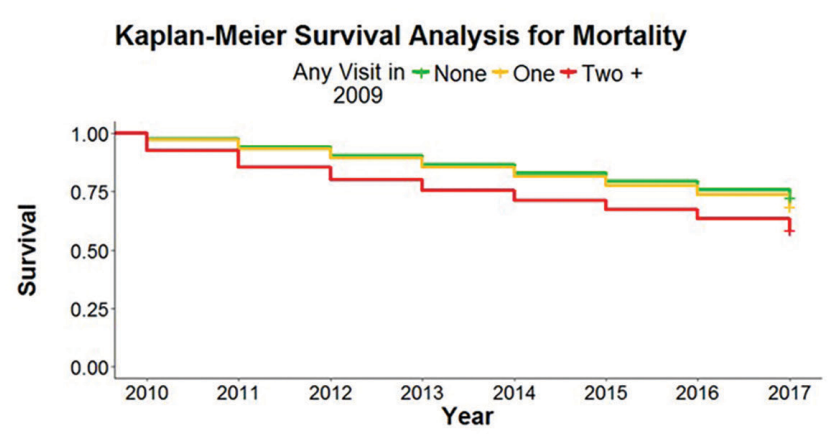

Figure 3 shows the Kaplan-Meier Survival Analysis between 2009 and 2014 based on any cause health care utilization in the Intermountain cohort (Figure 8A). Panel B shows the 8- year mortality for the VA cohort. The mortality curves separate for patients with no hospitalizations versus those with 2 or more, but any cause health care utilization does not separate mortality as well as severe COPD exacerbation frequency does.

data, mortality was significantly higher for the intermediate compared with infrequent health care utilizers (pairwise log rank test $p<0.001$ ). However, there was no statistical difference between 6-year allcause mortality in those intermediate and infrequent health care utilizers in the smaller cohort from the University of Chicago (Figure 3).

\section{Discussion}

In this study we examined the stability of severe COPD exacerbation frequency as a predictor of future COPD exacerbation frequency and overall mortality across 3 distinct clinical cohorts. Severe COPD exacerbation frequency is stable after 2 or more years of frequent exacerbations and predicts overall mortality in clinical 


\section{Figure 4. Charlson Comorbidity Rates According to Severe COPD Exacerbation Frequency}

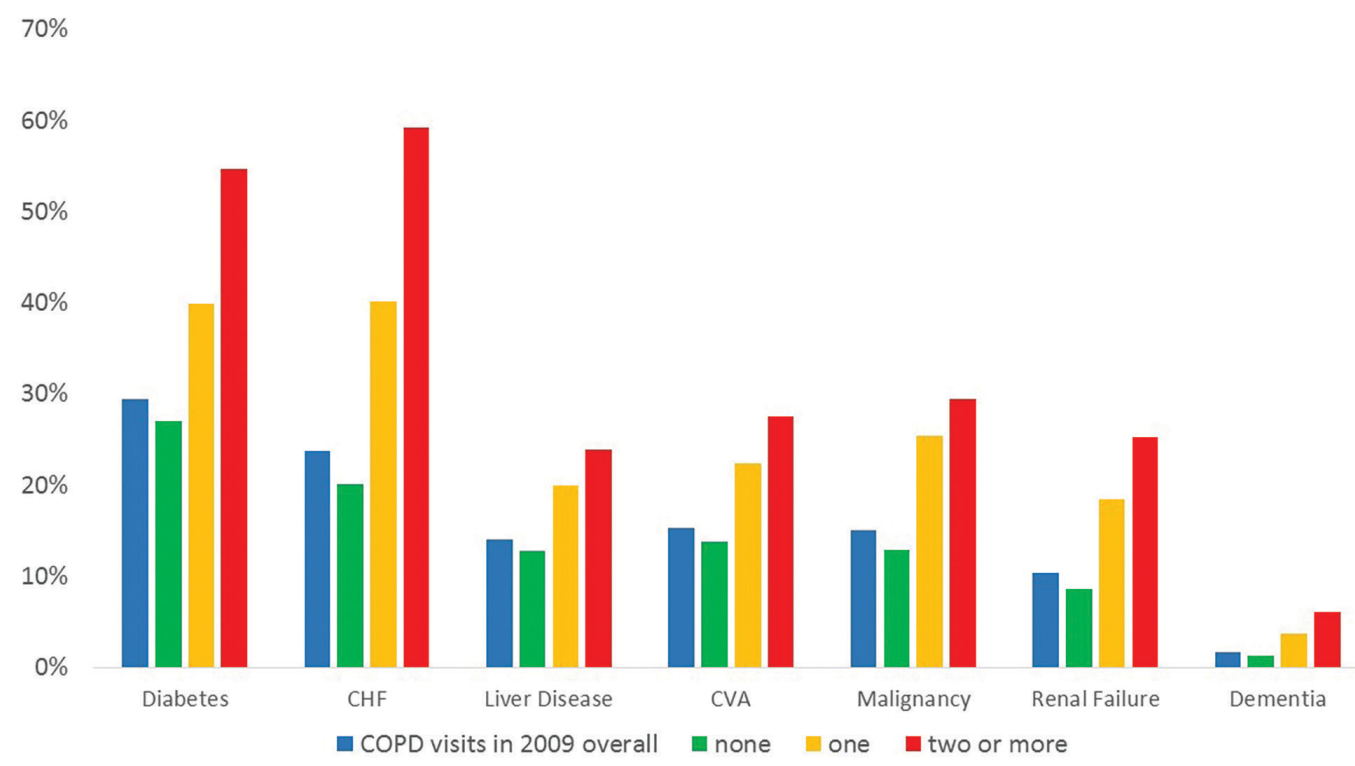

Figure 4 shows the Charlson Comorbidity rates according to COPD exacerbation frequency groups in the Intermountain Cohort. Frequent exacerbators have higher rates of cardiovascular morbidity (congestive heart failure, diabetes, renal failure and stroke) compared with non-exacerbators. Overall rates of comorbidity remain high for all groups.

\section{Figure 5. Severe COPD Exacerbation Frequency Stability Veterans Affairs}

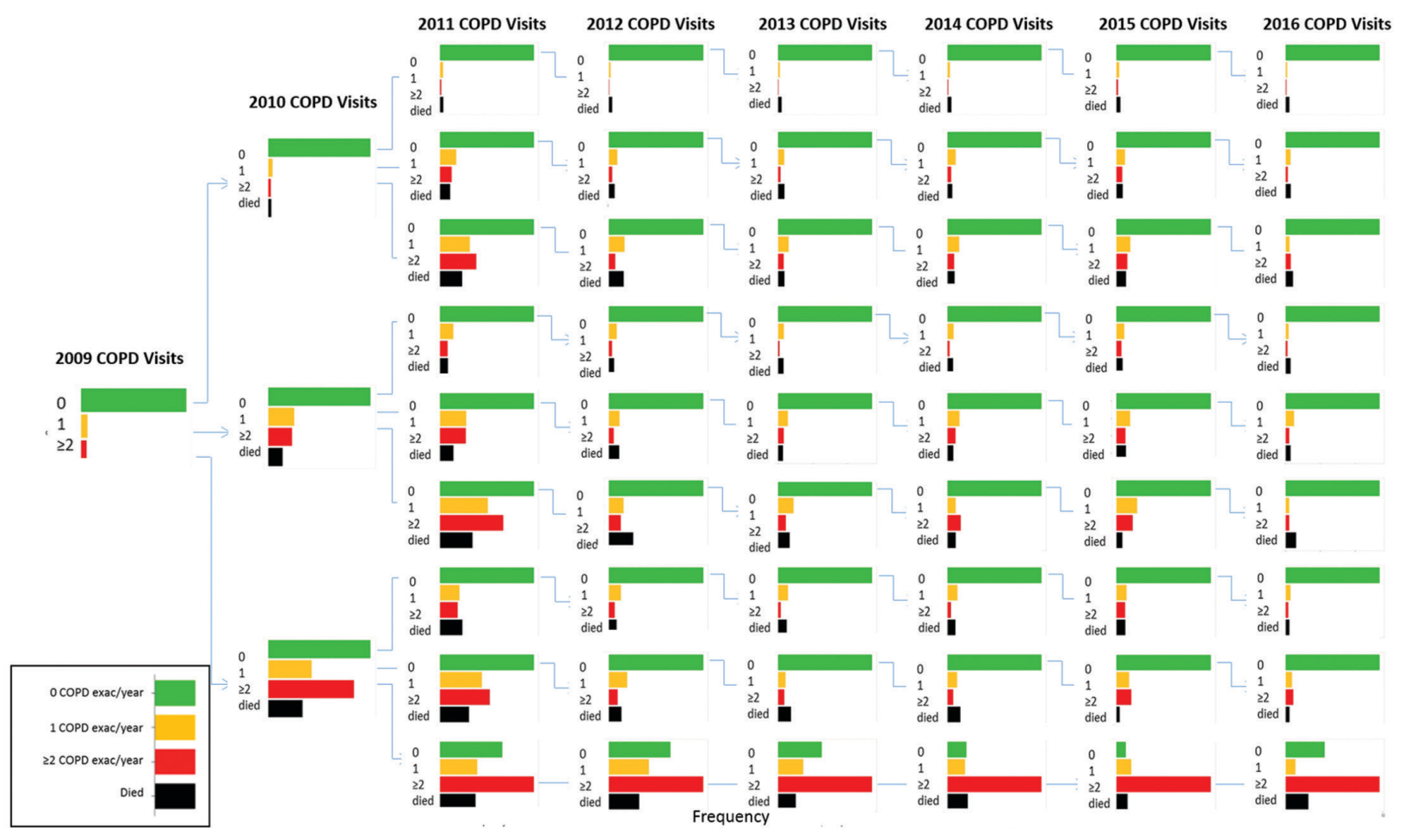

COPD exacerbation frequency for the Veterans Affairs (VA) cohort is shown over 8 years. Patients are divided into frequent (2 or more exacerbations per year), intermediate (1 exacerbation per year) and non-exacerbators (no exacerbations per year) in 2009 and then followed until 2016. Frequent exacerbators have a higher mortality than those with no exacerbations, and exacerbation frequency becomes stable within 2 years. 


\section{Figure 6. Severe COPD Exacerbation Frequency Stability University of Chicago Medicine System Cohort}

A.

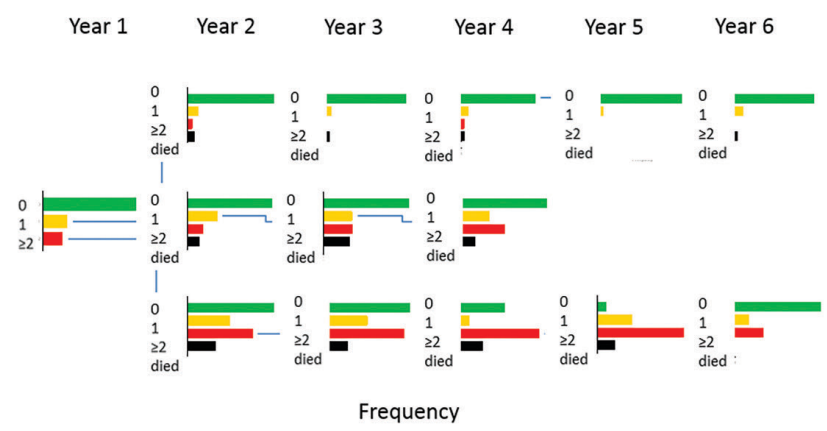

B.

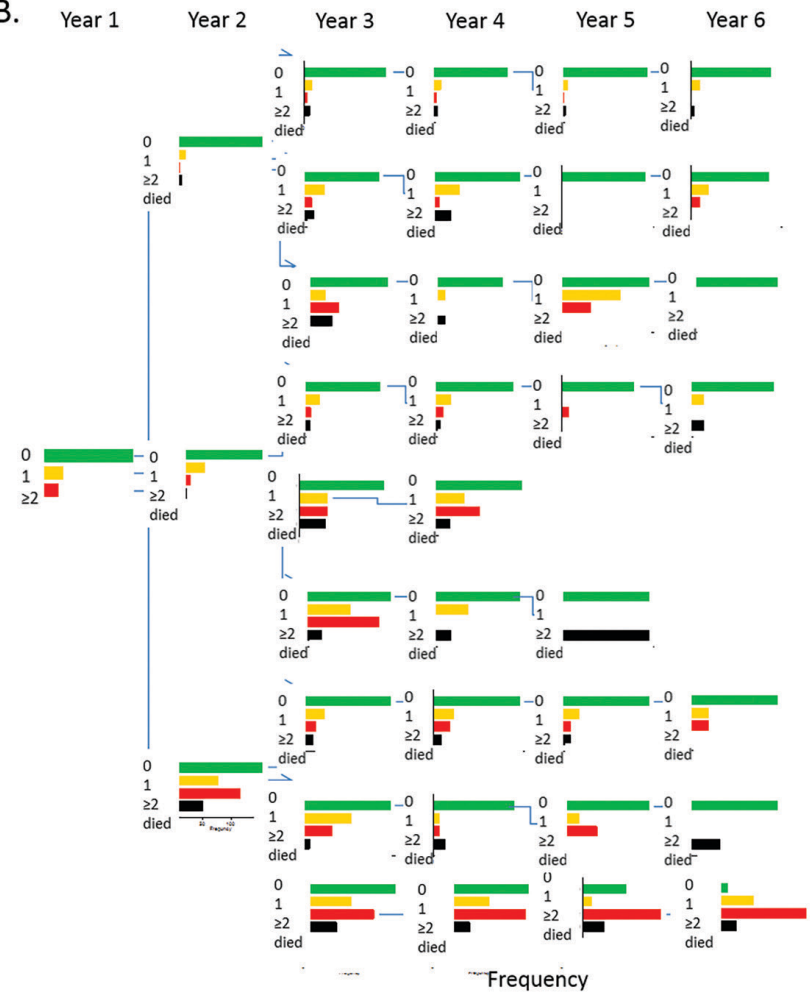

COPD exacerbation frequency for the University of Chicago Medicine system cohort is shown over 6 years. Patients are divided into frequent ( 2 or more exacerbations per year), intermediate ( 1 exacerbation per year) and non-exacerbators (no exacerbations per year) in 2009 and then followed until 2016. Panel A shows a simplified graph where patients were followed forward only if they remained stable. Panel B shows an extended graph where for the first 3 years patients were followed forward in all 3 categories. Non-exacerbator is a stable characteristic, whereas frequent exacerbators need to have frequent exacerbations for 4 consecutive years before becoming stable. This decrease in the stability of the frequent exacerbator phenotype in this open health system as compared with the other 2 relatively closed systems likely reflects an increased rate of hospitalizations outside the system for high risk patients.

cohorts identified by EHR data.

We also determined the stability of any cause health care utilization and its association with overall mortality. While overall health care utilization frequency is more stable than COPD exacerbation frequency, it does not predict overall mortality as well. Thus, while health care utilization-based metrics are imperfect and susceptible to well-described inaccuracies and inconsistencies in diagnosis, they appear to be reasonable starting points for identifying groups of patients of varying risks of future health care utilization (overall and COPD-specific), as well as overall mortality.

Our findings are in line with prior observations in recent studies that noted COPD exacerbation frequency is unstable unless present for 2 consecutive years. ${ }^{6}$
Our findings are in contrast to the ECLIPSE findings where, after 1 year, COPD exacerbation frequency was stable. ${ }^{3}$ The observed stability in the ECLIPSE cohort may reflect the fact that only patients who survived for all 3 years were included in the analysis. Furthermore, in contrast to clinical data, research studies for COPD patients have exclusion criteria (e.g., metastatic cancer), which may themselves contribute to health care utilization and overall mortality. $4,13,14$

Our study has several limitations. First, we identified patients with COPD based on billing codes. Thus, the accuracy of the COPD diagnosis and the presence and/or severity of the airflow obstruction were uncertain in our patients compared to individuals in research studies. ${ }^{3}$ However, health care utilization correlates with symptoms even in the absence of 


\section{Figure 7. Kaplan-Meier Survival by Severe COPD Exacerbation Frequency University of Chicago Cohort}

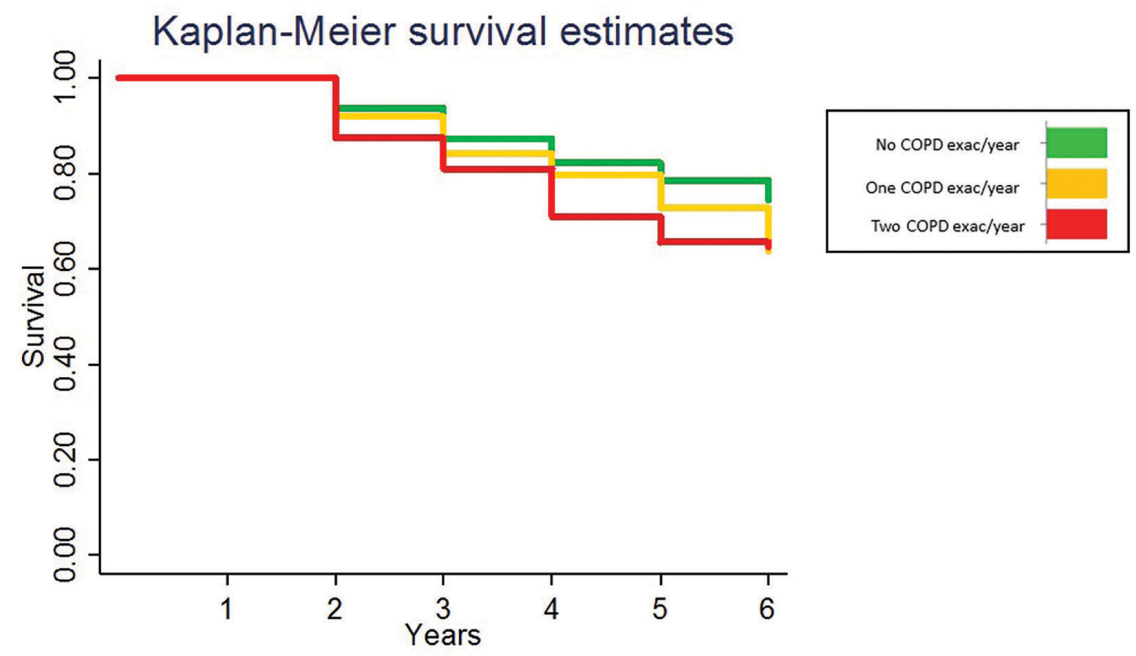

Figure 7 shows the 6-year Kaplan-Meier Survival Analysis for the University of Chicago Medicine system cohort based on COPD exacerbation frequency in the first year. Although there is a trend for lower mortality in for non-exacerbators compared with frequent exacerbators, these findings are not statistically significant.

obstruction, with updated GOLD guidelines relying on COPD exacerbation frequency and symptoms to categorize COPD patients. ${ }^{2,15}$ In addition, smokers with respiratory symptoms have health care utilization similar to patients with COPD and symptoms, and higher than patients with COPD without symptoms. ${ }^{16}$ Thus, health care systems looking to improve patient outcomes need new ways of identifying and categorizing former smokers with respiratory complaints. So, while lack of diagnostic certainty in our cohort is a limitation, it is reflective of the way patients with respiratory symptoms are treated clinically across health care systems.

As would be expected, our patients had high rates of cardiovascular comorbidities. These comorbidities create more diagnostic uncertainty when patients present with respiratory complaints. Indeed, many COPD trials exclude patients with significant heart failure. ${ }^{11}$ However, the high rate of cardiac comorbidity in our COPD patients is reflective of the complexity of patient care in a clinical setting.

Our health care utilization data captured only severe COPD exacerbations, namely those that resulted in an ED visit and/or hospitalization for COPD. Thus, milder COPD exacerbations treated in the outpatient setting or at home were not included. Additionally, while we included ED visits and hospitalizations in 2 relatively closed systems, we did not capture COPD exacerbations treated outside the health care systems studied. Our smallest cohort was in a relatively open health system at the University of Chicago Medicine system, where the small number of patients and likelihood of incomplete capture of clinical data with patients seeking care at multiple institutions likely contributed to the lack of mortality significance in this cohort.

In conclusion, the strength of our study is the evaluation of the stability of COPD exacerbation frequency, and health care utilization in general, in clinical data sets from 3 distinct health systems. Severe COPD exacerbation frequency was stable after 2 consecutive years and correlated with increased mortality across different health care systems. Thus, health care utilization (COPD-specific and all cause) may help identify patients who are at high risk for future health care utilization and overall mortality across a system. 


\section{Figure 8. Stability of Health Care Utilization Pattern for Any Cause Visits in the Intermountain Cohort}

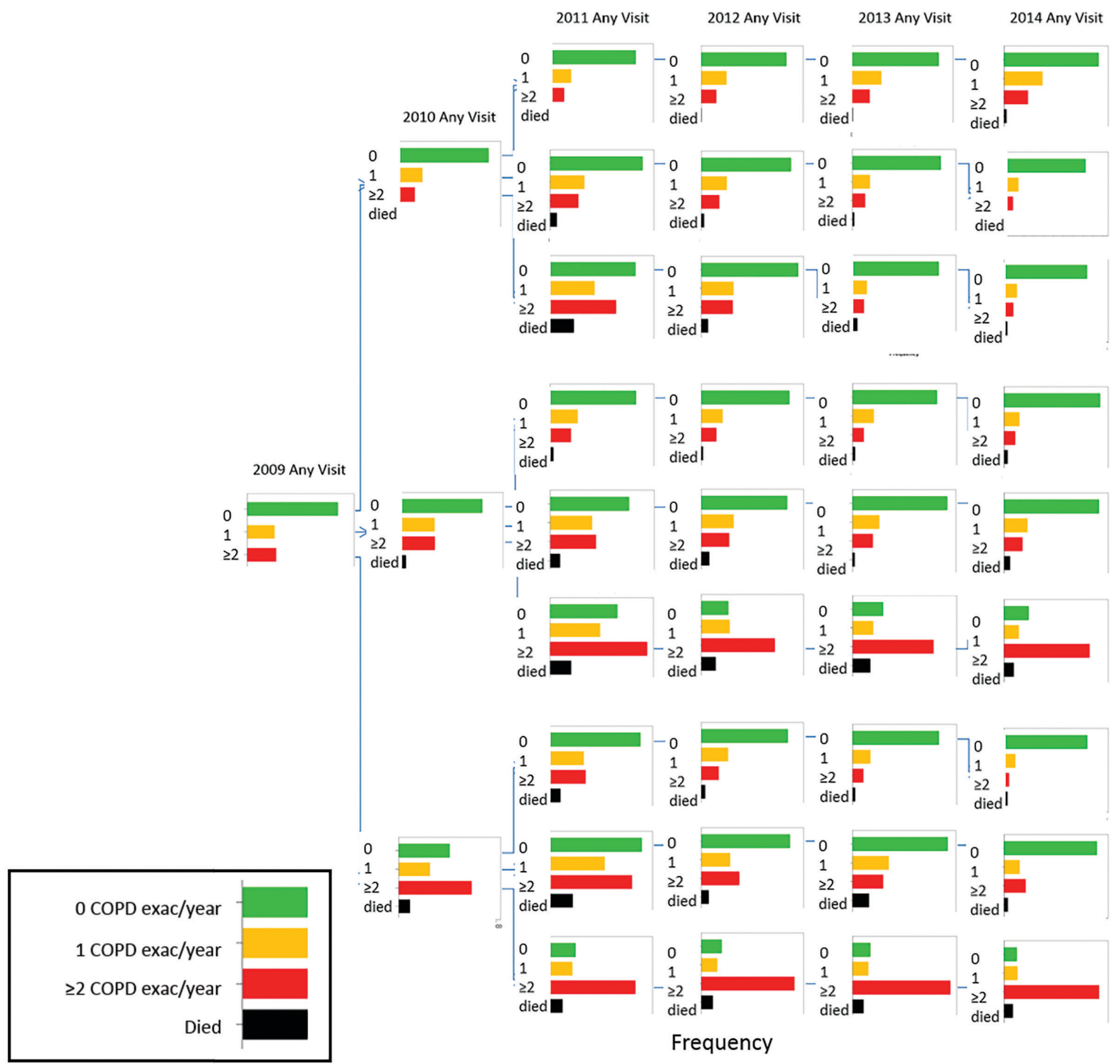

Any cause visit frequency for the Intermountain cohort is shown over 6 years. Patients are divided into frequent (2 or more visits for any cause per year), intermediate (1 visit per year) and non-exacerbators (no visits per year) in 2009 and then followed until 2014 . Any cause health care utilization is stable after just 1 year. High health care utilization ( 2 or more hospitalizations/ED visits for any cause) is a more stable characteristic than COPD exacerbations alone. 


\section{Figure 9. Stability of Health Care Utilization Pattern for Any Cause Visits in the Veterans Affairs Cohort}

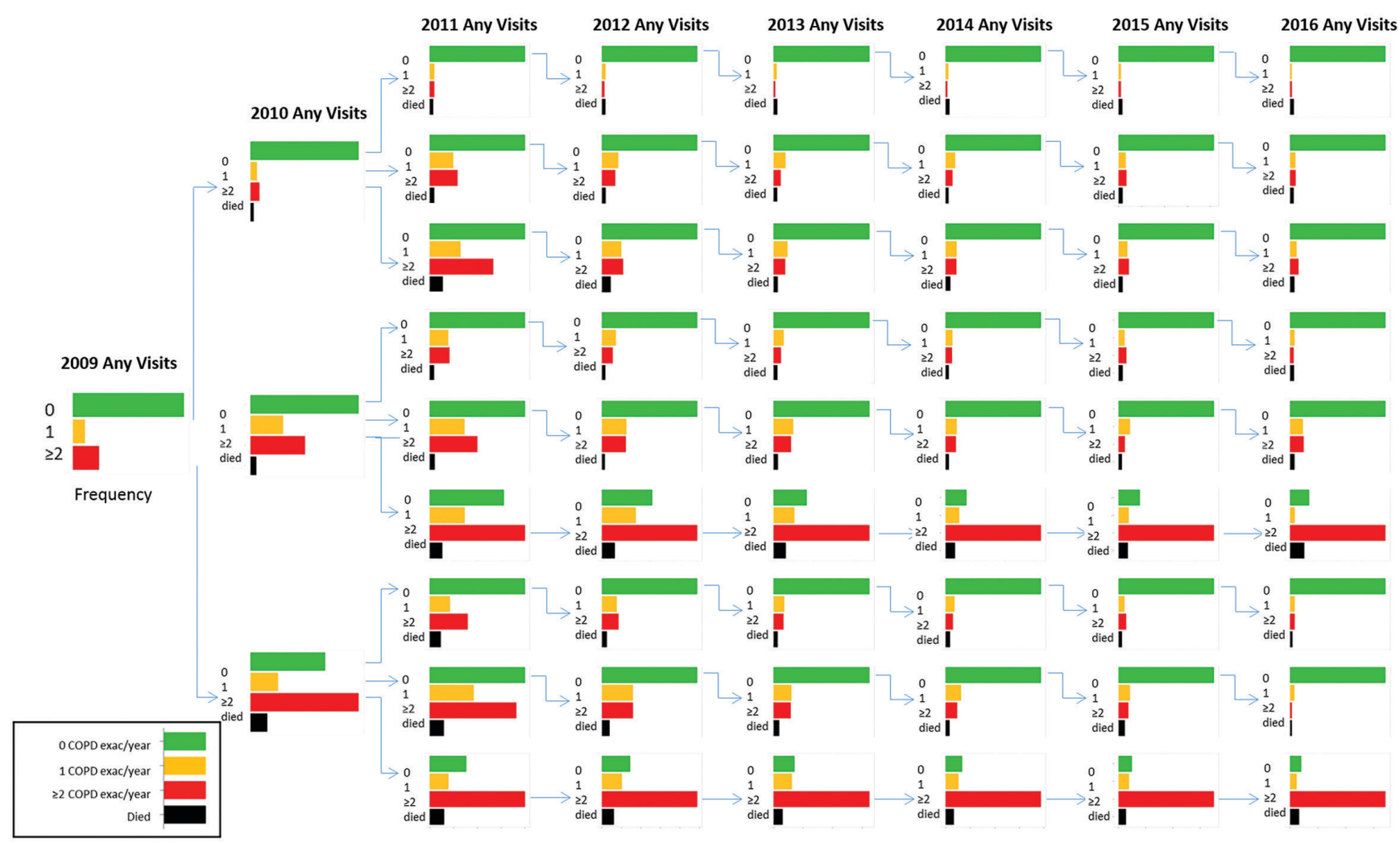

Any cause visit frequency for the Veterans Affairs (VA) cohort is shown over 8 years. Patients are divided into frequent (2 or more visits for any cause per year), intermediate (1 visit per year) and non-exacerbators (no visits per year) in 2009 and then followed until 2016. High health care utilization ( 2 or more hospitalizations /ED visits for any cause) is a more stable characteristic than COPD exacerbations alone.

\section{Acknowledgement}

Author contributions: D.B. conceived of the study, designed the data set, performed data analysis and interpreted the data, and wrote the first draft of the manuscript. D.C. helped design the study, performed the statistical analysis and validation, and edited the manuscript. S.R. generated and validated the dataset, helped analyze the data, and edited the manuscript. V.P., M.C. and K.C validated the findings in the University of Chicago data and edited the manuscript. R.M. assisted with data analysis and interpretation and edited the manuscript. S.Z. generated the data set and $\mathrm{R}$ code and validated the VA dataset and edited the manuscript. M.A. generated the data, analyzed the data for the validation in the VA cohort and edited the manuscript.

\section{Declaration of Interest}

The authors have no conflict of interest relevant to the manuscript. 


\section{References}

1. Vestbo J, Edwards LD, Scanlon PD, et al. Changes in forced expiratory volume in 1 second over time in COPD. New Eng $J$ Med. 2011;365:1184-1192.

doi: https://doi.org/10.1056/NEJMoa1105482

2. Global Initiative for Chronic Obstructive Lung Disease (GOLD). Global strategy for the diagnosis, management and prevention of COPD, 2017. GOLD website. http://goldcopd.org/gold-2017global-strategy-diagnosis-management-prevention-copd/ Published 2017. Accessed June 2018

3. Hurst JR, Vestbo J, Anzueto A, et al. Evaluation of CLtIPSEI. Susceptibility to exacerbation in chronic obstructive pulmonary disease. New Eng J Med. 2010;363:1128-1138. doi: https://doi.org/10.1056/NEJMoa0909883

4. Global Burden of Disease 2016 Disease and Injury Incidence and Prevalence Collaborators. Global, regional, and national incidence, prevalence, and years lived with disability for 328 diseases and injuries for 195 countries, 1990-2016: A systematic analysis for the global burden of disease study 2016. Lancet. 2017;390(10100):1211-1259.

doi: https://doi.org/10.1016/S0140-6736(17)32154-2

5. Soler-Cataluna J, Martínez-García MÁ, Sánchez PR, Salcedo E, Navarro M, Ochando R. Severe acute exacerbations and mortality in patients with chronic obstructive pulmonary disease. Thorax. 2005;60(11):925-931.

doi: https://doi.org/10.1136/thx.2005.040527

6. Han MK, Quibrera PM, Carretta EE, et al. Frequency of exacerbations in patients with chronic obstructive pulmonary disease: An analysis of the SPIROMICS cohort. Lancet Respir Med. 2017;5(8):619-626.

doi: https://doi.org/10.1016/S2213-2600(17)30207-2

7. Lawrence PJ, Kolsum U, Gupta V, et al. Characteristics and longitudinal progression of chronic obstructive pulmonary disease in GOLD B patients. BMC Pul Med. 2017;17:42. doi: https://doi.org/10.1186/s12890-017-0384-8

8. Reilev M, Lykkegaard J, Halling A, Vestbo J, Sondergaard J, Pottegard A. Stability of the frequent COPD exacerbator in the general population: A Danish nationwide register-based study. NPJ Prim Care Respir Med. 2017;27:25. doi: https://doi.org/10.1038/s41533-017-0029-7

9. Han MK, Agusti A, Calverley PM, et al. Chronic obstructive pulmonary disease phenotypes: The future of COPD. Am J Respir Crit Care Med. 2010;182:598-604. doi: https://doi.org/10.1164/rccm.200912-1843CC

10. Quan H, Sundararajan V, Halfon P, et al. Coding algorithms for defining comorbidities in ICD-9-cm and ICD-10 administrative data. Med Care. 2005;43:1130-1139. doi: https://doi.org/10.1097/01.mlr.0000182534.19832.83
11. Lee TA, Bartle B, Weiss KB. Spirometry use in clinical practice following diagnosis of COPD. Chest. 2006;129(6):1509-1515. doi: https://doi.org/10.1378/chest.129.6.1509

12. Connors Jr AF, Dawson NV, Thomas C, et al. Outcomes following acute exacerbation of severe chronic obstructive lung disease. The SUPPORT investigators (study to understand prognoses and preferences for outcomes and risks of treatments). Am J Respir Care Med. 1996;154(4):959-967. doi: https://doi.org/10.1164/ajrccm.154.4.8887592

13. Crisafulli E, Costi S, Luppi F, et al. Role of comorbidities in a cohort of patients with COPD undergoing pulmonary rehabilitation. Thorax. 2008;63:487-492. doi: https://doi.org/10.1136/thx.2007.086371

14. Fabbri L, Luppi F, Beghe B, Rabe K. Complex chronic comorbidities of COPD. Eur Respir J. 2008;31(1):204-212. doi: https://doi.org/10.1183/09031936.00114307

15. Woodruff PG, Barr RG, Bleecker E, et al. Clinical significance of symptoms in smokers with preserved pulmonary function. New Eng J Med. 2016;374:1811-1821. doi: https://doi.org/10.1056/NEJMoa1505971

16. Woodruff PG, Barr RG, Bleecker E, et al. Clinical significance of symptoms in smokers with preserved pulmonary function. New Eng J Med. 2016;374:1811-1821. doi: https://doi.org/10.1056/NEJMoa 1505971 